http://dx.doi.org/10.21611/qirt.2017.060

\title{
THE EVALUATION OF BIMATERIAL SPECIMEN WITH FDM 3D PRINTING TECHNOLOGY BY INFRARED THERMOGRAPHY
}

\author{
Gwang-Wook Hong ${ }^{1 \dagger}$, Hong-Geun Park', Gang-Min Lim² ${ }^{2}$ Joo-Hyung Kim ${ }^{1 *}$ \\ ${ }^{1}$ Department of Mechanical Engineering, Inha University, Inha-ro 100, Nam-gu, Incheon 22212, Republic of Korea \\ ${ }^{2}$ Asset Technology Group, \#803, Seongnam-daero 345, Bundang-gu, Seongnam-si, Gyeonggi-do, Republic of Korea \\ †Presenting Author: gw.hong@inha.edu \\ ${ }^{*}$ Corresponding Author: joohyung.kim@inha.ac.kr
}

\begin{abstract}
The evaluation of temperature variation is very important issue relations in the fields of mechanical design, maintenance and advanced material. Development of carbon fiber or variable composites material is increasingly used in novel material especially aerospace field. One of the key factors in developing novel materials is finding alternative sources of power. Recent research trend is to develop self-actuating device from natural source. The main example is shape memory alloys(SMA), shape memory polymer(SMP) and etc. To develop the SMA or SMP, Thermal properties of material are important. Then the use of nondestructive testing(NDT) techniques is useful to get thermal data about novel materials. Infrared thermography(IRT) is convenient and useful method of NDT technique. However, IRT require various variables to get a proper data. In this research, we trying to reduce the number and find the emissivity of variables on bimaterial from 3D printing.
\end{abstract}

KEYWORDS: Active infrared thermography, Bimaterial, FDM, 3D Printing, Uncooled infrared camera, Composites material, NDT, Quantitative analysis.

\section{INTRODUCTION}

3D printing is technology used for the rapid production of 3D objects directly using digital computer aided design (CAD) files. [1] 3D printing methods are Stereo lithography(SLA), Inkjet Printing, Selective Laser Sintering (SLS), Fused Deposition Modeling (FDM) and Laminated Object Manufacturing (LOM) etc. [2] Above all, FDM is very cheap and widely used. As 3D printer is used as general, Researchers are paying to thermal problems. In this regard, Various temperature measurement systems like thermocouples and resistance temperature detectors (RTD) are in general contact type and does not provide a visual image of the object under investigation. One of a novel NDT method, Infrared thermography (IRT) measures the temperature of a body remotely and provides very convenient and intuitive monitoring techniques. IRT is available to various fields such as Monitoring of civil structures, Monitoring of electrical and electronic components, Deformation monitoring, Inspection of machineries, Corrosion monitoring, Weld monitoring, Application of IRT in nuclear industries, IRT based condition monitoring in aerospace industries, composites materials and other application etc. [3] Most of all, active measurement methods are using an external light source to measure the emission ratio of ingredients and to develop actuator like shape memory polymer(SMP) element. In this research, using the active infrared thermography can understand basic physical properties of the composites and bimaterial. Details explanation will be given below.

\section{EXPERIMENTAL}

For thermal evaluation of bimaterial specimen, bimaterial was made of composites polymer by the 3D printing technology. After the fabrication, the bimaterial specimen was moved to thermal measuring setup. Depending on the temperature change, the sample are measured by IR-detector.

\subsection{FABRICATION FOR TEST SPECIMEN USING 3D PRINTER}


The 3D printer used was a double-head and x-y type FDM. It was used nylon/carbon fiber composites and nylon based on Polylactic acid(PLA) filaments. Usually FDM 3D printing used Acrylonitrile butadiene styrene(ABS), however ABS was bad for the health better than PLA. For this reason, bimaterial was fabricated PLA. The complete 3D printed bimaterial specimen is presented in Fig. 1.

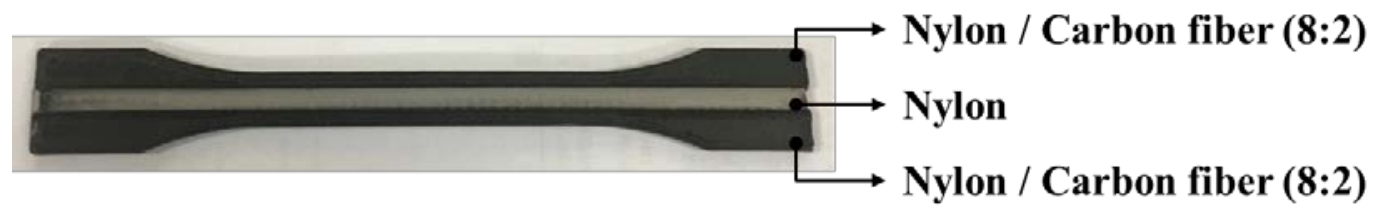

Fig. 1 Bimaterial specimen using ISO 527-2 standard.

\subsection{SET OF THE INFRARED THERMOGRAPHY MEASUREMENT}

The measurement setup consist of IR-detector, Thermometer, Hotplate and Control PC. IR-detector was performed with a FLIR T650SC uncooled infrared camera operating in the long-wave band (7.5-13.0 um), with a Vanadium Oxide(VOx) sensor and a 640 x 480 pixel image format. The temperature range of the IR-detector was $-40 \sim+150{ }^{\circ} \mathrm{C}$. It can record radiometric IR sequences in real-time directly on the camera to control PC. These sequences contain all temperature data and can be post analyzed on the PC. Thermometer used the thermocouple(TC) that is connect to Digital-multimeter(DMM). TC was used to measure the real temperature on the surface of hotplate. Hotplate was directly heat source for bimaterial specimens. Measuring data from IR-detector transferred the Control PC. Control PC can save and post processing the data. Fig. 2 shows measurement setup for temperature gradient for bimaterial.

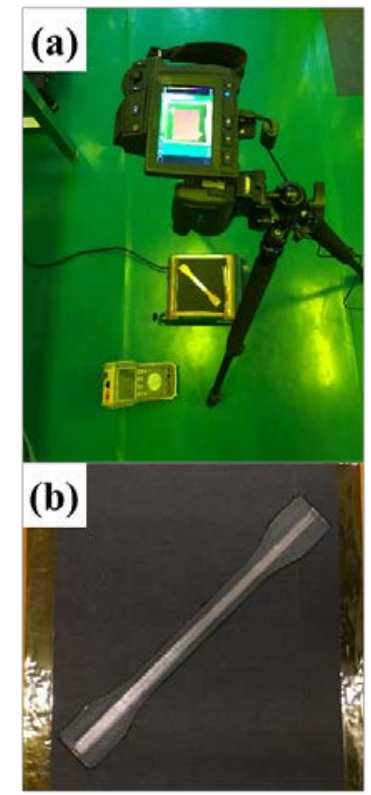

(c)

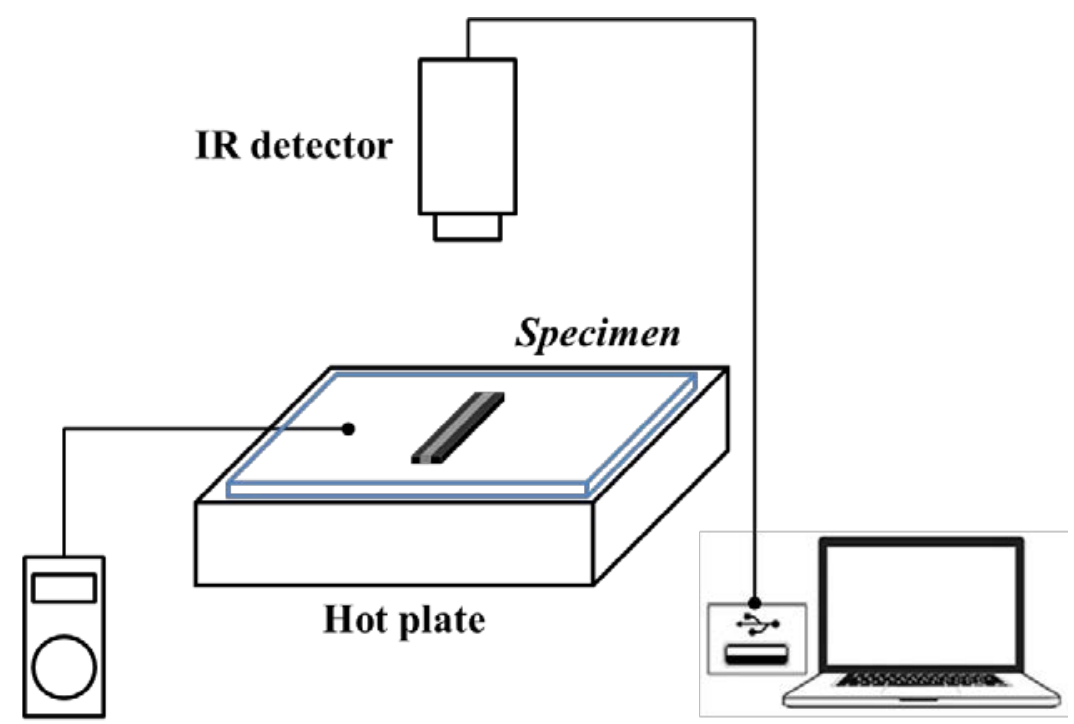

Thermocouple
(Thermometer)

Control PC

Fig. 2 The measurement setup (a) Real image for measuring system, (b) Location of bimaterial specimen on the hotplate, (c) Diagram for measuring system.

\section{RESULTS AND CONCLUSIONS}

The evaluation of thermal properties for bimaterial by using sensitive uncooled IR-detector present in Fig. 3. When the temperature of the hot plate rises, place the specimen up and measured the specimen 3 minutes later. At this point, the temperature of hotplate was set to $40,60,80$ and $100{ }^{\circ} \mathrm{C}$. To obtain the proper data of each specimen, hotplate surface was 
covered thick black paper except the specimen area and level / span range match with others. As a result, the higher the heat source temperature, the temperature variation of all specimens can be clearly observed.

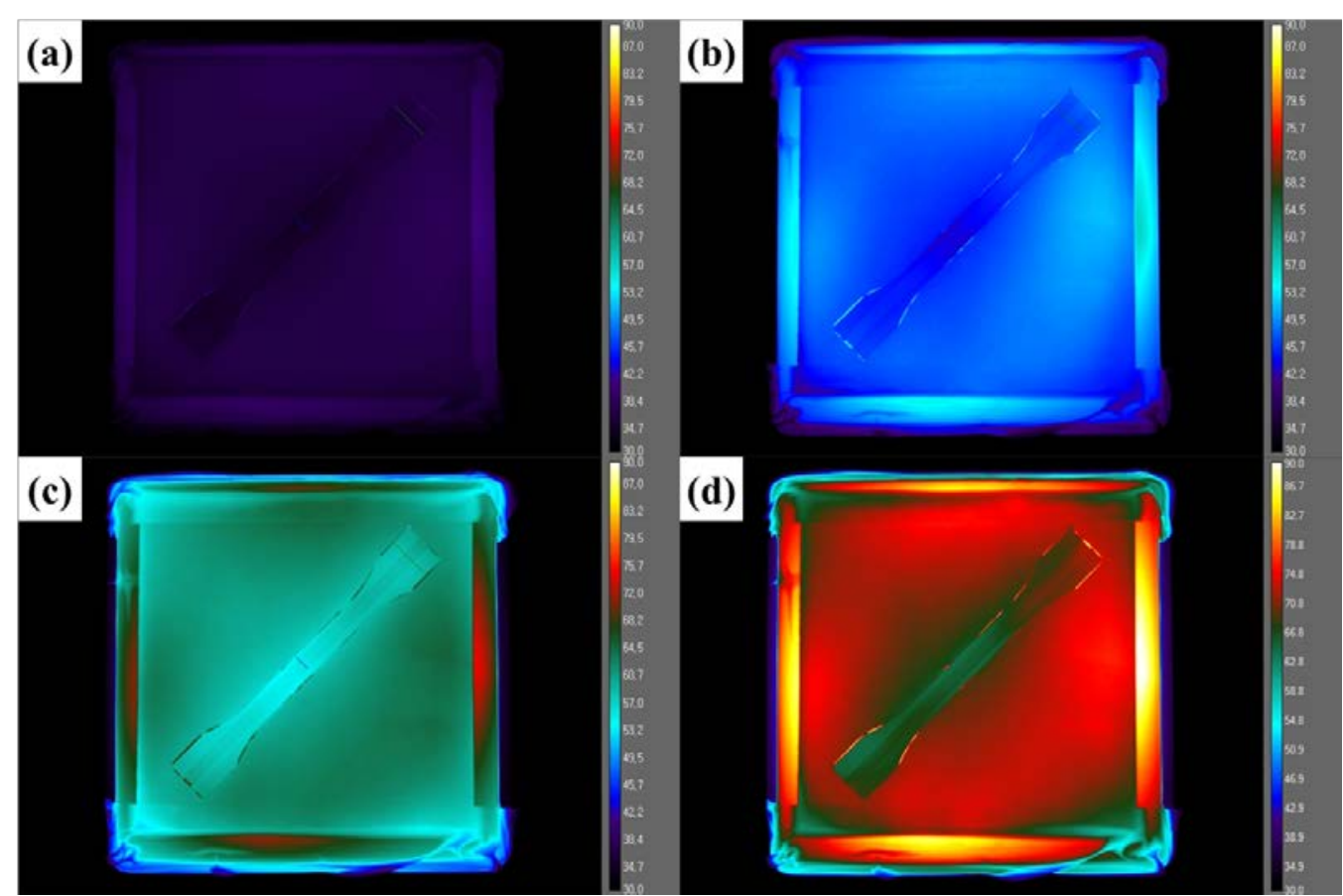

Fig. 3 A temperature variations of bimaterial put on hotplate after 3 min and setting temperature of hotplate (a) $40{ }^{\circ} \mathrm{C}$, (b) $60{ }^{\circ} \mathrm{C}$, (c) $80^{\circ} \mathrm{C}$, (d) $100^{\circ} \mathrm{C}$.

The emissivity was calculated as the ratio of measured values of TC and IR-detector, with a value of 0.9. IRT monitoring clearly observed the different condition. The covering unnecessary heat sources could be restrained to IR-detector. In addition this research will be measuring the various material and need to use the other heat source instead of hotplate.

\section{ACKNOWLEDGMENT}

This work was supported by Institute for Information \& communications Technology Promotion(IITP) grant funded by the Korea government(MSIP) (No.2016-0-00452(R7520-16-0005), Development of creative technology based on complex 3D printing technology for labor, the elderly and the disabled).

\section{REFERENCES}

[1] S.J. Leigh, R.J. Bradley, C.P. Pussell, D.R. Billson, D.A. Hutchins, A simple, low-cost conductive composite material for 3D printing of electronic sensors, PloS one. 7 (2012) e49365.

[2] B.C. Gross, J.L. Erkal, S.Y. Lockwood, C.P. Chen, D.M. Spence, Evaluation of 3D printing and its potential impact on biotechnology and the chemical sciences, ANALYTICAL CHEMISTRY. 86 (2014) 3240-3253.

[3] S. Bagavathiappan, B.B. Lahiri, T. Saravanan, J. Philip, T. Hayakumar, Infrared thermography for condition monitoring-a review, Infrared Physics \& Technology. 60 (2013) 35-55. 